\title{
ORBITS IN STRIPS
}

\author{
JAROSLAV ZEMÁNEK \\ Institute of Mathematics, Polish Academy of Sciences \\ P.O. Box 21, 00-956 Warszawa, Poland \\ E-mail: zemanek@impan.gov.pl
}

By a strip we mean a subset of the complex plane that is bounded by two parallel lines.

Let $A$ be a bounded linear operator on a Hilbert space such that, for each fixed unit vector $x$, the scalar-valued orbit

$$
\left\{\left\langle A^{n} x, x\right\rangle: n \in \mathbb{N}\right\}
$$

is contained in some strip (depending on $x$ ). Does it follow that all orbits (1), for all unit vectors $x$, are contained in a common strip?

If the space is finite-dimensional, we can associate with $A=\left(a_{i j}\right)$ the Gerschgorin set

$$
\mathcal{G}(A)=\bigcup_{i}\left\{z \in \mathbb{C}:\left|a_{i i}-z\right| \leq \sum_{j \neq i}\left|a_{i j}\right|\right\} .
$$

Suppose that the spectral radius of $A$ is no more than 1 , and that all $\mathcal{G}\left(A^{n}\right)$, for $n \in \mathbb{N}$, are contained in a fixed strip. Does it follow that the powers $A^{n}$ are bounded, for all $n \in \mathbb{N}$ ?

The above questions are motivated by the paper [1].

\section{References}

[1] A. Gomilko, I. Wróbel, and J. Zemánek, Numerical ranges in a strip, in: Operator Theory 20, K. R. Davidson, D. Gaşpar, Ş. Strătilă, D. Timotin, and F.-H. Vasilescu (eds.), Proceedings of the 20th International Conference on Operator Theory, Timişoara (Romania), June 30-July 5, 2004, Theta, Bucharest, 2006, 111-121. 